\title{
A Study on Edge Detection Techniques in Retinex Based Adaptive Filter
}

\author{
P. Swarnalatha and Dr. B. K. Tripathy
}

\begin{abstract}
Processing the images to obtain the resultant images with challenging clarity and appealing visualization are the major challenges. So, pre-processing of images is required to meet these challenging needs. In many applications like rendering high dynamic range images, certain pre-processing is necessary. This kind of pre-processing includes detecting the high contrast edges, smoothing the noise using filters etc. In this paper, the main focus is on the application of edge detection in rendering high dynamic range (HDR) images using Retinex-based adaptive filter method. Different edge detection techniques are used in rendering HDR images using Retinex algorithm and an observation has been made that Canny edge detection yields visually appealing resultant image.
\end{abstract}

Index Terms-High Dynamic Range, Edge Detection, Retinex

\section{INTRODUCTION}

HDR image rendition using Retinex-based adaptive filter method is experimentally studied as shown in Fig-1. In this method, the image will undergo Principal Component Analysis (PCA) to obtain luminance and chrominance components. Retinex operation is only applied on luminance channel in which the edge detection is used to find the mask of the high contrast edges in the image. The mask is used in the computation of Retinex operation and the edge detection plays a vital role in this computation $[1,2]$. Edges in images are areas with strong intensity contrasts. The set of connected pixels that lie on the boundary between two regions is also an edge [1]. Edge detection refers to the process of identifying and locating sharp discontinuities in a gray scale image [4]. It significantly reduces the amount of data and filters out useless information, while preserving the important structural properties in an image. The discontinuities are abrupt changes in pixel intensity which characterize the boundaries of objects in a scene. Classical methods of edge detection involve convolving the image with an operator. Optics, sampling and other image acquisition imperfections yield edges that are blurred. The degree of blurring is determined by factors such as the quality of the image acquisition system, the sampling rate and illumination conditions under which the image is acquired [3]. There is extremely large number of edge detection operators available which are specific to certain type of edges. Variables involved in the selection of an edge detection operator include:

- Edge Orientation: The geometry of the operator determines a characteristic direction in which it is most

P. Swarnalatha, VIT University, Vellore,India, email id:pswarnalatha@vit.ac.in, Member, IACSIT

Dr. B. K. Tripathy, VIT University, Vellore, India, email id: tripathybk@vit.ac.in sensitive to edges. Operators can be optimized to look for horizontal, vertical or diagonal edges.

- Noise environment: Edge detection is difficult in noisy images, since both the noise and the edges contain high-frequency content. Operators used on noisy images are typically larger in scope, so they can average enough data to reduce the localized noisy pixels which results in less accurate localization of the detected edges.

- Edge Structure: Effects such as refraction, poor focus can result in objects with boundaries defined by a gradual change in intensity. In such a case, operators need to be chosen to be responsive to such a gradual change in intensity.

There are many ways to perform edge detection. However, the majority of different methods may be grouped into two categories [2, 3].

Gradient: The gradient method detects the edges by looking for the maximum and minimum in the first derivative of the image. The magnitude of the first derivative can be used to detect the edge at a point in an image. In second derivatives, zero-crossings may occur and so the sign of the second derivative can be used to determine whether an edge pixel lies on the dark or light side of an edge. The second derivative is more sensitive to noise. So, image smoothing is to be considered prior to the use of derivatives in finding the edge pixels of an image. A point is said to be an edge point if the two-dimensional first order derivative exceeds the threshold chosen at that point. The set of such points connected is defined as an edge. In second order derivatives, the edge points are defined if there are zero-crossings [2].

\section{Gradient Operators}

First-order derivatives of a digital image are based on various approximations of the 2-D gradient. The gradient of an image $f(x, y)$ at location $(x, y)$ is defined as the vector

$$
\nabla f=\left[\begin{array}{l}
G_{x} \\
G_{y}
\end{array}\right]=\left[\begin{array}{l}
\frac{\partial f}{\partial x} \\
\frac{\partial f}{\partial y}
\end{array}\right]=\left[G_{x}^{2}+G_{y}^{2}\right]^{1 / 2} \approx\left|G_{x}\right|+\left|G_{y}\right|
$$

where $\nabla f$ is the gradient, $G_{x}$ and $G_{y}$ are the magnitudes of gradient in vertical and horizontal directions respectively. The gradients of various gradient based edge detection operators are discussed in later sections.

Laplacian: The Laplacian method searches for zero crossings in the second derivative of the image to find the edges and to highlight the location of the image. The Laplacian of a 2-D function $f(x, y)$ is a second order derivative defined as 


$$
\nabla^{2} f=\frac{\partial^{2} f}{\partial x^{2}}+\frac{\partial^{2} f}{\partial y^{2}}
$$

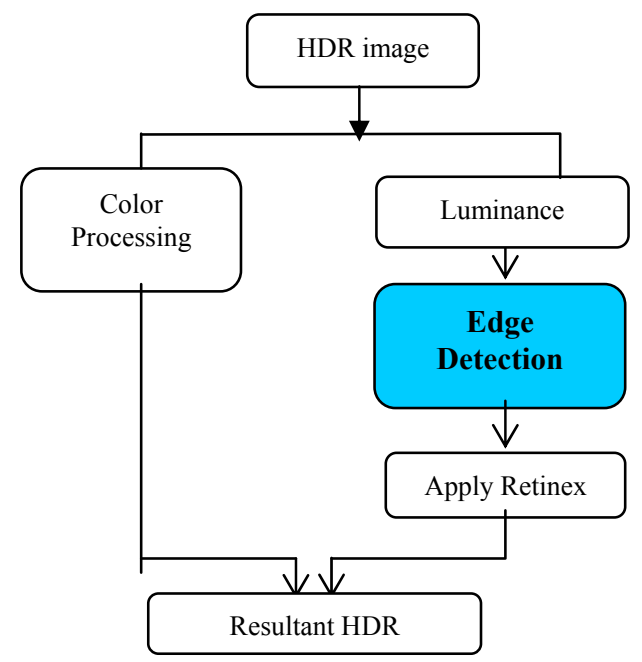

Fig-1. Application of edge detection in Retinex-Based Adaptive Filter method

\section{Edge Detection TechniQues}

Sobel Edge Detector: The gradient at the centre point in a neighbourhood is computed as follows by the Sobel detector

$$
G=\left[G_{x}^{2}+G_{y}^{2}\right]^{1 / 2}
$$

where $G$ is the magnitude of the gradient, $G_{x}$ and $G_{y}$ are the magnitudes of gradient in vertical and horizontal directions respectively. Then, we say that a pixel at location $(x, y)$ is an edge pixel if $g \geq T$ at that location, where $T$ is a specified threshold. Typically, an approximate magnitude of the gradient can be computed using $\left|G_{x}\right|+\left|G_{y}\right|$ which is much faster to compute. The angle of orientation of the edge giving rise to spatial gradient is [5]

$$
\theta=\tan ^{-1}\left(\frac{G_{y}}{G_{x}}\right)
$$

Prewitt Edge Detector: This method is similar to Sobel method and is used to detect vertical and horizontal edges in images. Prewitt detector is slightly simpler to implement computationally than the Sobel detector, but it tends to produce some noisier results.

Robert Edge Detector: The Roberts edge detector uses the masks as shown in Figure 1 to approximate digitally the first derivatives $G_{x}$ and $G_{y}$. Roberts's detector is one of the oldest edge detectors in digital image processing and it is also simplest. This detector is used considerably less than other methods due to its limited functionality (e.g., it is not symmetric and cannot be generalized to detect edges that are multiples of $45^{\circ}$ ). The gradient magnitude is

$$
\left|G_{x}\right|+\left|G_{y}\right|
$$

The angle of orientation of the edge giving rise to spatial gradient is

$$
\theta=\tan ^{-1}\left(\frac{G_{y}}{G_{x}}\right)-3 \Pi / 4
$$

Laplacian of Gaussian (LoG) Detector: The Laplacian is a 2-D isotropic measure of the second spatial derivative of an image. The Laplacian of an image highlights regions of rapid intensity change and is therefore often used for edge detection. The Laplacian is often applied to an image that has been smoothed with something approximating a Gaussian Smoothing filter in order to reduce its sensitivity to noise. The operator normally takes a single gray level image as input and produces another gray level image as output.

The Laplacian $\boldsymbol{L}(\boldsymbol{x}, \boldsymbol{y})$ of an image with pixel intensity values $\boldsymbol{I}(\boldsymbol{x}, \boldsymbol{y}) \mathrm{I}(\mathrm{x}, \mathrm{y})$ is [1]

$$
L(x, y)=\frac{\partial^{2} I}{\partial x^{2}}+\frac{\partial^{2} I}{\partial y^{2}}
$$

Since the input image is represented as a set of discrete pixels, we have to find a discrete convolution mask that can approximate the second derivatives in the definition of the Laplacian. The image is Gaussian smoothed before applying the Lapalacian filter to reduce the noise. This pre-processing step reduces the high frequency noise components prior to differentiation step. The 2-D LoG function centered on zero and with Gaussian standard deviation $u$ has the form [4]

$$
\begin{aligned}
& \operatorname{LoG}(x, y)=-\frac{1}{\Pi \sigma^{4}}\left[1-\frac{r^{2}}{2 \sigma^{2}}\right] e^{\frac{r^{2}}{2 \sigma^{2}}} \\
& \text { where } r^{2}=x^{2}+y^{2}
\end{aligned}
$$

Zero Crossings detector: The zero crossing detector looks for places in the Laplacian if an image where the value of the Laplacian passes through zero i.e., points where the Laplacian changes sign. This detector returns a binary image with single pixel thickness lines showing the position of the zero-crossings in image which lie on closed contours.

Canny: There are so many techniques to detect the edges of an image as mentioned above of which Canny edge detection method is the optimal one. Canny followed a list of criteria to improve current methods of edge detection. The first and the most obvious is low error rate. It is important that edges occurring in images should not be missed and that there be NO responses to non-edges. The second criterion is that the edge points be well localized. In other words, the distance between the edge pixels as found by the detector and the actual edge is to be at a minimum. A third criterion is to have only one response to a single edge. This was implemented because the first two were not substantial enough to completely eliminate the possibility of multiple responses to an edge. Based on these criteria, the canny edge detector first smoothes the image to eliminate and noise. It then finds the image gradient to highlight regions with high spatial derivatives. The algorithm then tracks along these regions and suppresses any pixel that is not at the maximum (non-maximum suppression). The gradient array is now further reduced by hysteresis. Hysteresis is used to track along the remaining pixels that have not been suppressed. Hysteresis uses two thresholds and if the magnitude is below the first threshold $\boldsymbol{T} \mathbf{1}$, it is set to zero (made a non-edge). If the magnitude is above the high threshold $\boldsymbol{T} 2$, it is made an edge. And if the magnitude is between the two thresholds, then it is set to zero unless there is a path from this pixel to a pixel with 
gradient above $\boldsymbol{T} \mathbf{2}$.

Canny's edge detection method can be summarized as follows $[3,4]$ :

1. The image is smoothed using a Gaussian filter with a specified standard deviation $\sigma$, to reduce noise.

2. The local gradient $\boldsymbol{g}(\boldsymbol{x}, \boldsymbol{y})=\left[\boldsymbol{G}_{x}^{2}+\boldsymbol{G}_{y}^{2}\right]^{1 / 2}$, and edge direction, $\alpha(x, y)=\tan ^{-1}\left(\boldsymbol{G}_{y} / \boldsymbol{G}_{x}\right)$ are computed at each point. Any of the three techniques Sobel, Prewitt and Roberts can be used to compute $\boldsymbol{G}_{\boldsymbol{x}}$ and $\boldsymbol{G}_{\boldsymbol{y}}$. An edge point is defined to be a point whose strength is locally maximum in the direction of the gradient.

3. The edge points determined in (2) give rise to ridges in the gradient magnitude image. The algorithm then tracks along the top of these ridges and sets to zero all pixels that are not actually on the ridge top so as to give a thin line in the output, a process known as non-maximal suppression. The ridge pixels are then thresholded using two thresholds, $\boldsymbol{T} \mathbf{1}$ and $\boldsymbol{T} \mathbf{2}$, with $\boldsymbol{T} \mathbf{1}<\boldsymbol{T} \mathbf{2}$. Ridge pixels with values greater than $\boldsymbol{T} \mathbf{2}$ are said to be "strong" edge pixels. Ridge pixels with values between $\boldsymbol{T 1}$ and $\mathbf{T 2}$ are said to be "weak" edge pixels.

4. Finally, the algorithm performs edge linking by incorporating the weak pixels that are 8-connected to the strong pixels.

\section{Performance Evaluation Metrics}

In order to evaluate the performance of the above mentioned edge detection algorithms, mean and standard deviation are used for justification.

Standard Deviation: It is known that standard deviation is composed of the signal and noise parts. This metric would be more efficient in the absence of noise. It measures the contrast in the image. An image with high contrast would have a high standard deviation.

$$
S D=\sqrt{\sum_{i=0}^{L}(i-\bar{i})^{2} h_{I_{f}}(i)}, \bar{i}=\sum_{i=0}^{L} i h_{I_{f}}
$$

where $h_{I_{f}}(i)$ is the normalized histogram of the image.

\section{COMPARISON OF Edge Detection TeChNiQueS}

Gradient-based algorithms such as Prewitt detector are prone to major drawback of being very sensitive to noise. These algorithms have masks and coefficients of fixed size and are adaptable to fixed noise levels. Among the Laplacian based edge detectors, Canny's algorithm performs better in most of the scenarios. The performance of the Canny algorithm depends heavily on the adjustable parameters, $\sigma$ which is standard deviation of the Gaussian filter and threshold values $\boldsymbol{T 1}$ and $\boldsymbol{T 2}$. The bigger the value for $\sigma$, the larger the size of the Gaussian filter becomes. This implies more blurring, necessary for noisy images, as well as detecting larger edges. Smaller values of $\sigma$ imply a smaller Gaussian filter which limits the amount of blurring, maintaining finer edges in the image. Larger the scale of Gaussian, less accurate is the localization of the edge. Canny edge detector performs better compared to gradient based algorithms also.
The following images below are the observations which prove that Canny edge detection algorithm performs better. Weak edges are detected only if they are connected to the strong edges. The Sobel, Prewitt and Robert algorithms detect the strong edges only but whereas the Canny algorithm detects the strong and weak edges [6].

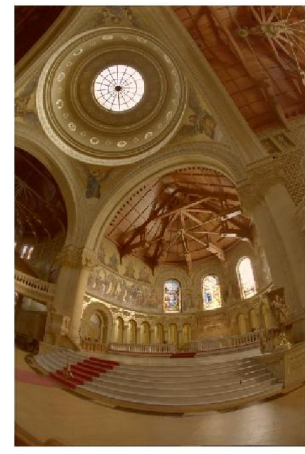

Fig-2. Original Image

Various edge detection operators are applied to the Fig-2 without specifying any thresholds and sigma values and are displayed below.

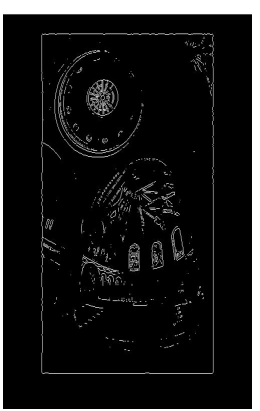

(a)

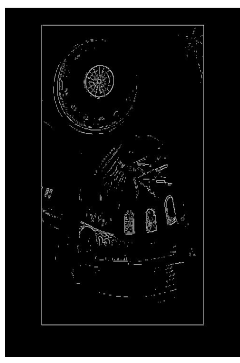

(c)

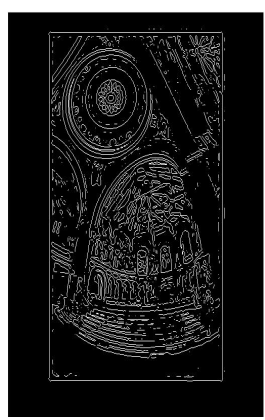

(e)

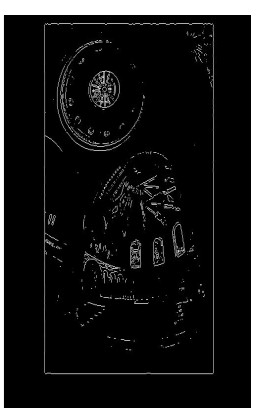

(b)

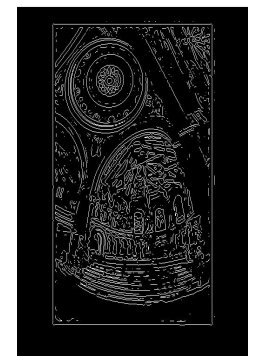

(d)

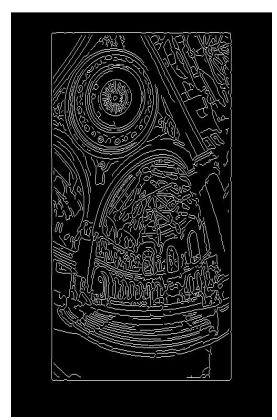

(f)
Fig-3. Edge detections of Fig-2 

(a) Sobel edge detection
(b) Prewitt edge detection
(c) Robert edge detection
(d) LoG edge detection
(e) Zero-crossing edge detection
(f) Canny edge detection

In Fig-3, the results of various edge detection algorithms on Fig-2 are shown. One can observe that there is no much detail in Sobel, Prewitt and Robert operators. In LoG and Zero-crossing edge detections, the discontinuity is more and the edge pixels are not connected properly. But in the Canny edge detection, there is detailed visibility and the week edges connected to the strong edges can be observed. In Table-1, the threshold parameter is set to 0.1 and the various edge detection algorithms are performed on the Fig-2 and mean, standard deviation and time are computed. One can observe that the mean and the standard deviation are maximum for the Canny edge detector indicating that the edge detection is maximum. The computational complexity is also less for the Canny edge detection comparatively to the gradient based edge detectors (Sobel, Prewitt and Robert). The execution time is less for the LoG and Zero-crossing edge detectors but the edge detection is not clear as per the observation. In the similar manner, the in Table-2, the threshold parameter is set to 0.1 and the various edge detection algorithms are performed on the Fig-2 and mean, standard deviation and time are computed. Even in this case, the mean and the standard deviation are maximum for Canny and it is better than the other edge detection algorithms. Taking the execution time into consideration, the Canny detects the edges faster as per the observation. As mentioned before, the mean and standard deviation of the resultant HDR image of Retinex based adaptive filter method are computed and shown in Table-3. The mean is maximum for Canny and the standard deviation is less as per the observation from the below tables and the graphs but the Canny yields the resultant image with good visualization.

TABLE 1: MEAN, STANDARD DEVIATION AND TIME COMPUTATIONS FOR FIG-1 USING VARIOUS EDGE DETECTION OPERATORS WITH THRESHOLD $=0.1$.

\begin{tabular}{|c|c|c|c|}
\hline $\begin{array}{c}\text { Edge detection } \\
\text { operator }\end{array}$ & Mean & $\begin{array}{c}\text { Standard } \\
\text { deviation }\end{array}$ & Time (sec) \\
\hline Sobel & 0.0149 & 0.1212 & 0.644 \\
\hline Prewitt & 0.0146 & 0.1200 & 0.696 \\
\hline Robert & 0.0145 & 0.1193 & 0.647 \\
\hline LoG & 0 & 0 & 0.177 \\
\hline Zero-crossing & 0 & 0 & 0.166 \\
\hline Canny & 0.238 & 0.1706 & 0.238 \\
\hline
\end{tabular}

TABLE 2: MEAN, STANDARD DEVIATION AND TIME COMPUTATIONS FOR FIG-1 USING VARIOUS EDGE DETECTION OPERATORS WITH THRESHOLD $=0.5$.

\begin{tabular}{|c|c|c|c|}
\hline $\begin{array}{c}\text { Edge detection } \\
\text { operator }\end{array}$ & Mean & $\begin{array}{c}\text { Standard } \\
\text { deviation }\end{array}$ & Time (sec) \\
\hline Sobel & 0.0004 & 0.0070 & 0.644 \\
\hline Prewitt & 0 & 0 & 0.643 \\
\hline Robert & 0.0012 & 0.0349 & 0.651 \\
\hline LoG & 0 & 0 & 0.163 \\
\hline Zero-crossing & 0 & 0 & 0.163 \\
\hline Canny & 0.0070 & 0.0837 & 0.176 \\
\hline
\end{tabular}

TABLE 3: MEAN AND STANDARD DEVIATION OF THE RESULTANT IMAGE OF FIG-1AFTER APPLYING RETINEX ALGORITHM WITH VARIOUS EDGE DETECTION OPERATORS.

\begin{tabular}{|c|c|c|}
\hline Edge detection operator & Mean & $\begin{array}{c}\text { Standard } \\
\text { deviation }\end{array}$ \\
\hline Sobel & 0.2833 & 0.1509 \\
\hline Prewitt & 0.2833 & 0.1509 \\
\hline Robert & 0.2829 & 0.1510 \\
\hline LoG & 0.2803 & 0.1516 \\
\hline Zero-crossing & 0.2803 & 0.1516 \\
\hline Canny & 0.2869 & 0.1505 \\
\hline
\end{tabular}

The above tables are graphically represented in figures 4-7. The selection of threshold value is a essential consideration and so the mean, standard deviation and time of varying thresholds are graphically plotted and compared and an observation has been made that as the threshold increases, mean and standard deviation also increases and the time also increases. From these observations, it has been concluded that the threshold value should not be too high or too low. As the maximum threshold is 1 and to detect the high contrast edges, the threshold can be chosen in between 0.1 and 0.5 in order to improve the local contrast.

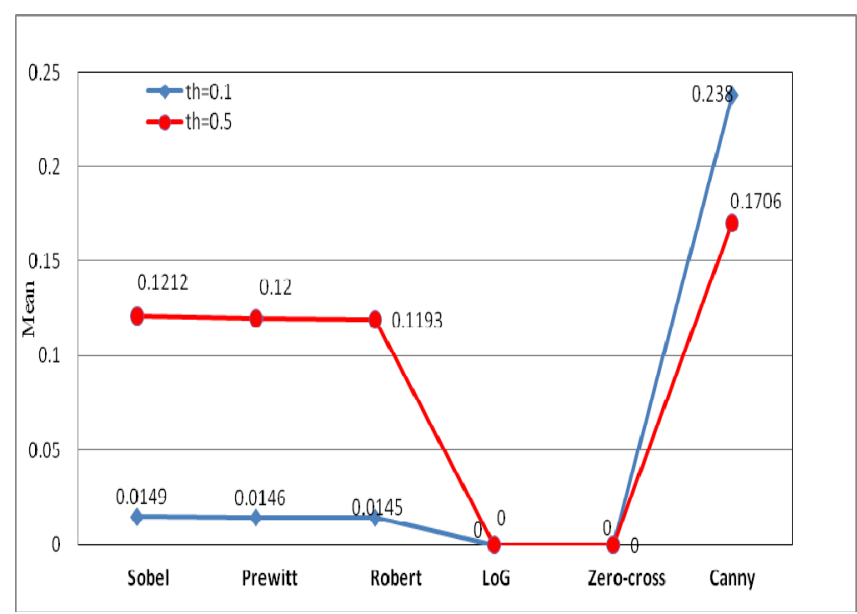

Fig-4. Mean for Fig-1 using various edge detection operators with threshold $=0.1$ and threshold $=0.5$ as shown in Tables 1 and 2 .

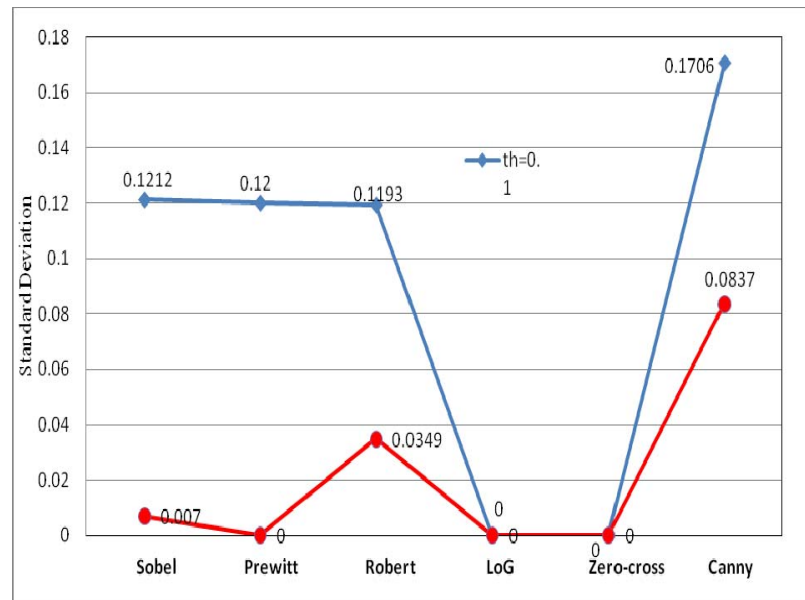

Fig-5. Standard Deviation for Fig-1 using various edge detection operators with threshold $=0.1$ and threshold $=0.5$ as shown in Tables 1 and 2 . 


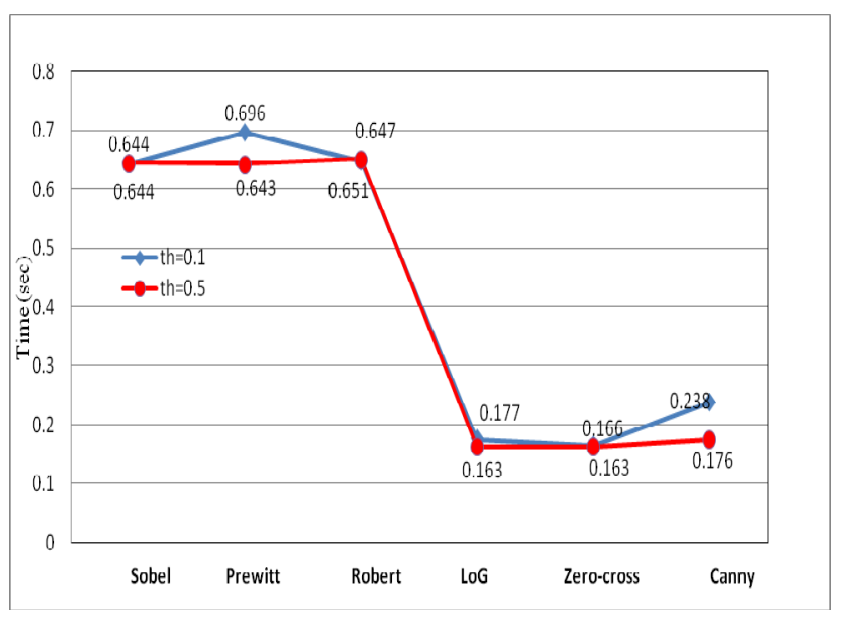

Fig-6. Computational time for Fig-1 using various edge detection operators with threshold $=0.1$ and threshold $=0.5$ as shown in Tables 1 and 2 .

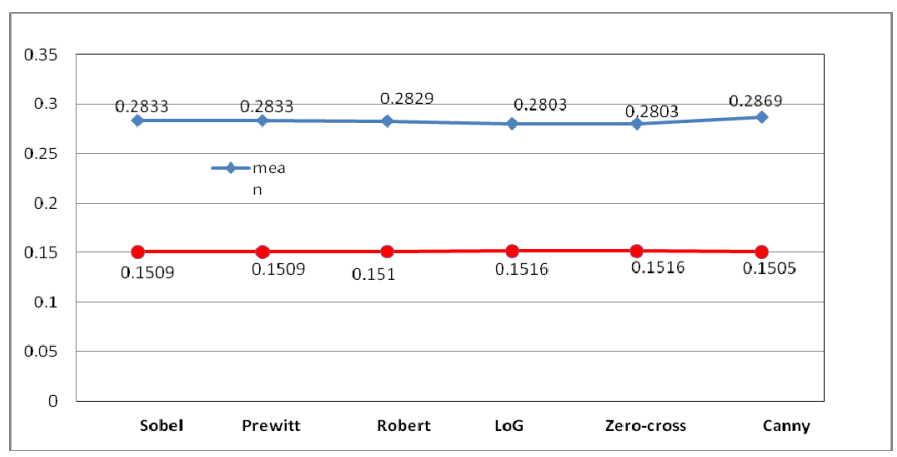

Fig-7. Mean and Standard Deviation (SD) for Fig-1 after applying Retinex using various edge detection operators as shown in Table-3.

\section{CONCLUSION}

Different edge detection techniques were studied and it is concluded that Canny method performed very well at cast of execution time. All these edge detection algorithms were used in Retinex based adaptive filter to compute the mask which is used in Retinex operation and evaluated. As expected, Canny edge detection performed well in computation of mask and hence good visually appealing image can be rendered using the Retinex operation with the help of Canny edge detector.

\section{ACKNOWLEDGMENT}

I take this opportunity to convey my regards to the guide for the entire guidance for preparation of this paper as part of the research work. I thank the ISRO authorities and VIT administration for providing support to the research.

\section{REFERENCES}

[1] Laurence Meylan and Sabine Susstrunk, "High dynamic range image rendering with a Retinex based adaptive filter", IEEE Trans. On Image Proc., vol.15, No.09, pp.2820-2830, Sep. 06.

[2] Rafael C. Gonzalez, Richard E. Woods, "Digital Image Processing", Pearson Education, Inc. and Dorling Kindersley, Fourth Impression, 2007, pg no. $594-607$.

[3] Rafael C. Gonzalez, Richard E. Woods, Steven Eddins, "Digital Image Processing Using MATLAB", Pearson Education, Inc. and Dorling Kindersley, Fourth Impression, 2008, pg no. 398 - 407.
[4] J.Canny, "A computational approach to edge detection," IEEE Trans Pattern Anal.Mach. Intell.,vol. PAMI-8, no.6, pp. 679-698, Jum.1986.

[5] Advanced edge detection technique: Techniques in computational vision,

"http://www.cpsc.ucalgary.ca/Research/vision/501/edgedetect.pdf"

[6] Bill Green, "A tutorial on edge detection techniques", http://www/pages.drexel.edu/ weg22/can_tut.html, 2002

Swarnalatha Purushotham, member of IACSIT, Vellore, 04.11.77. M.Tech (CSE), VIT, Vellore, Pursuing Ph.D (Intelligent Systems). Assistant Professor ( $\mathrm{Sr}$ ), in the school of computing sciences and engineering, VIT University, at Vellore, India, has published more than 15 papers and guided many students of UG and PG so far. She is having 10 years of teaching experiences. She is associated with CSI, ACM, IACSIT, IEEE(WIE). Her current research interest includes Image Processing, Neural Networks, Pattern Recognition, Remote Sensing.

B.K Tripathy, a senior professor in the school of computing sciences and engineering, VIT University, at Vellore, India, has published more than hundred papers and guided ten students for $\mathrm{PhD}$. so far. He is having more than 28 years of teaching experiences. His name has come out in Marquis silver jubilee edition of who's who in the world (USA) and also in the International Biographical Centre, Cambridge (London). He is associated with CSI, IMS, OITS,OMS, IACSIT, IEEE and many other professional bodies. He is also associated as an editor with many national and international journals. His current research interest includes knowledge engineering, fuzzy systems, list theory and social networks. 